\title{
Role of Biquad and Hilbert Filters in Removing Noise - A Simulink Analysis
}

\author{
Animesh Kumar Jha \\ Tata Consultancy Services \\ Mumbai, India
}

\begin{abstract}
Often some noise signals get interleaved with speech signals and owing to noisy surroundings the captured signal may not sound as desired. For such cases, a combination of Hilbert transform filter and Biquad filter in cascaded form might prove helpful. An arrangement in a simulink model demonstrates successful noise reduction in a voice signal recorded from a microphone. Both the filters used were of Direct Form structure, while the Hilbert filter was a Direct Form FIR, the Biquad was Direct Form II structure filter. The observations for speech signal as well as noise signals have been produced as results that show a significant noise removal.
\end{abstract}

Keywords: Biquad filter; noise; Simulink

\section{Introduction}

Noise signals when combine with a useful signal, reduce the desirability of that signal. It may result from the unwanted signals present in the surroundings but mostly its contribution reduces/hides the information present in the actual signal. There are various ways for detecting and removing such signals. Typical methods include hamming code for noise detection and correction as well. Better methods (for large data size) include filtering using a combination of low pass and high pass filters. Sometimes an anti-aliasing filter must also be used when data is transmitted from a distance as the high frequency signals acquire the identity of low frequency signals.

But in conditions where the noise removal is considered only on a workstation, where no data long distance transmission comes into play, the filtering technique is extremely useful over its other counterparts. These filters include FIR filters, lowpass, high pass, bandpass, etc and IIR filters. FIR filters also find their use in decimation, a method used to reduce the number of elements in an input sequence and it merges every certain number of $\mathrm{N}$ samples into one.

Some techniques also use distributed filtering theory for prediction, but its essence has grown weak over the years with the development of adaptive filtering theory [8-10] where filters adapt themselves according to the input signal. In this paper, filters in cascaded form have been used and signals captured have also been shown to demonstrate their effects. Observations have also been performed on Rayleigh and Band Limited White noise to examine the performance of the filter structure.

The Hilbert Transform of a signal results in a +90 degree shift of signal's negative frequency components, and a -90 degree shift of its positive frequency components. It can be said that Hilbert Transform calculates the analytic signal and eliminates the carrier quite well. Hilbert Transform relates the real part and the imaginary part of any physically viable linear time invariant system which implies a system should be stable as well as causal. 


\section{Simulink Components Used}

The filters used in the model were Hilbert Filter and Biquad Filter. The usefulness of biquad filters can be seen in [1-4] while that of Hilbert filters can be seen in [5]. The design considerations for a biquad filter can also be obtained from [11]. A test signal was produced in a noisy surrounding and corresponding waveforms were captured In figure 2, the waveform shown (Time Scope 1) is the source signal, while the waveform shown in Figure 3 is a noise corrected signal. The difference in the signals can be observed easily by looking at the peaks of the source waveform over some period of time and then observing the output waveform for the same period. The input signal after 1 second till 2 second can be seen and it can be easily inferred that the signal consists of mostly noise, as no signal was transmitted to the mic after 1 second for the next 1 second. The same signal when observed in the output signal was corrected and showed a relatively lesser rise in amplitude.

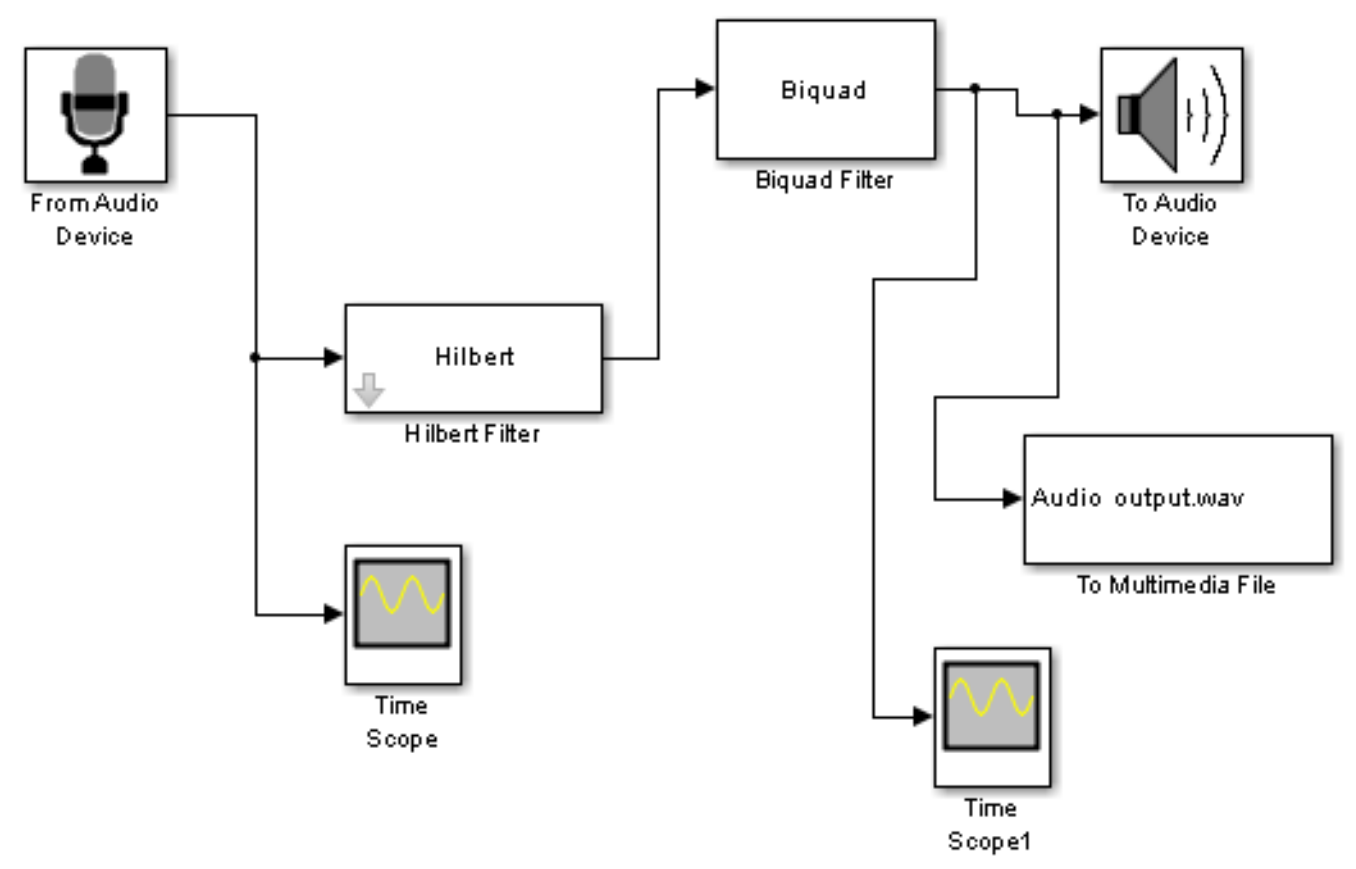

Figure 1. Block Diagram of the Simulink Model

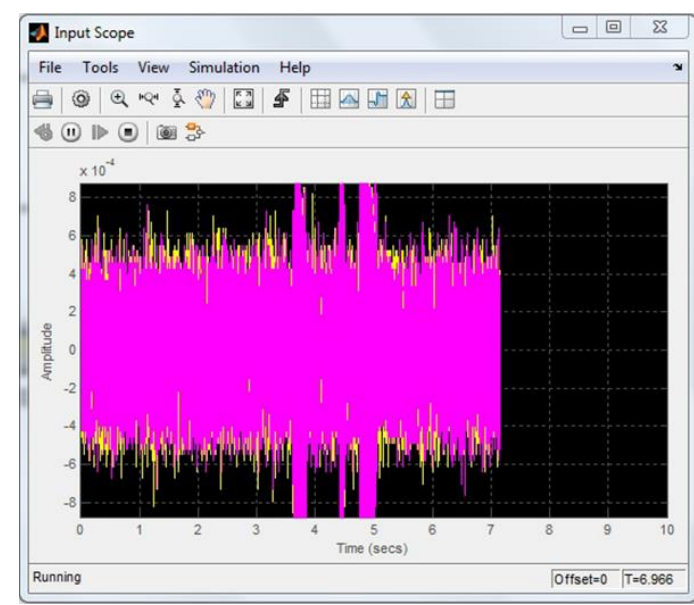

Figure 2. Test Signal Input 


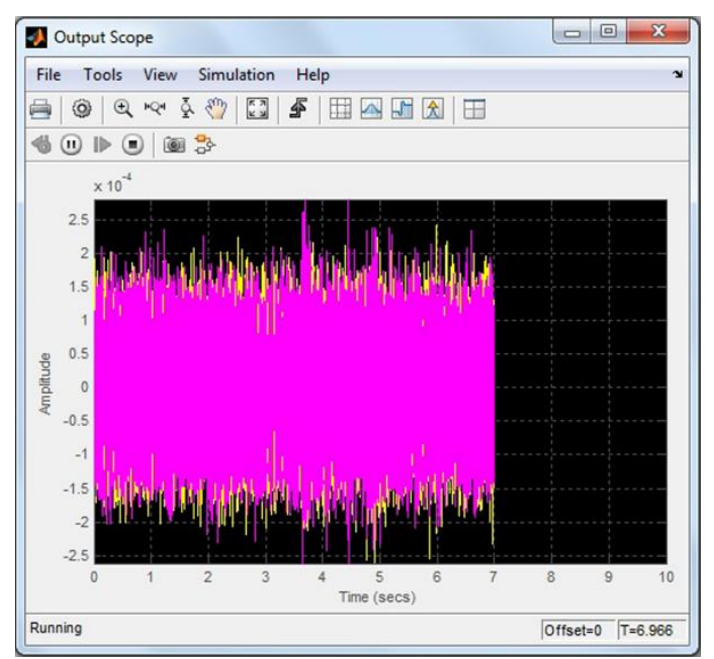

Figure 3. Test Signal Output

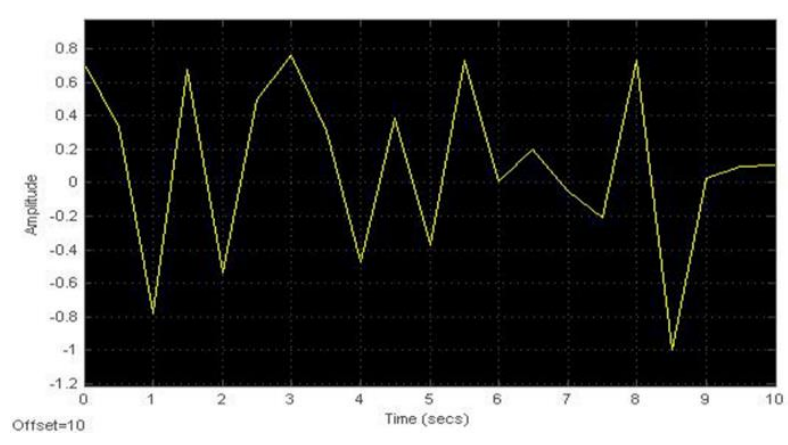

Figure 4. Band Limited White Noise Input

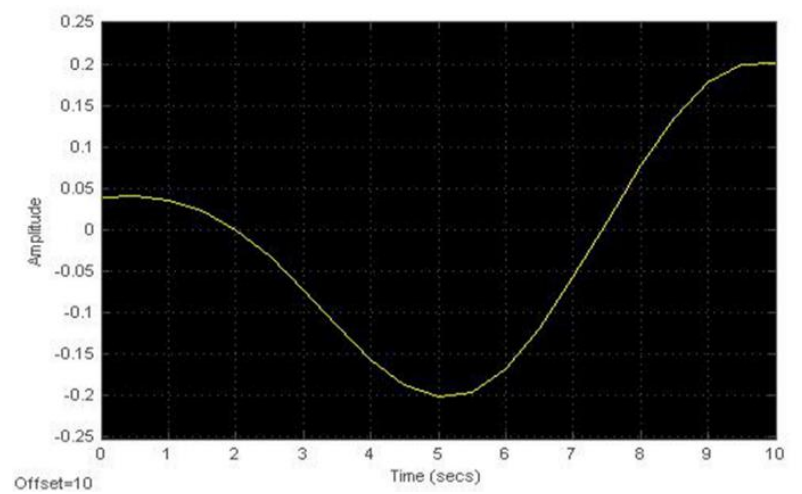

Figure 5. Band Limited White Noise Output 


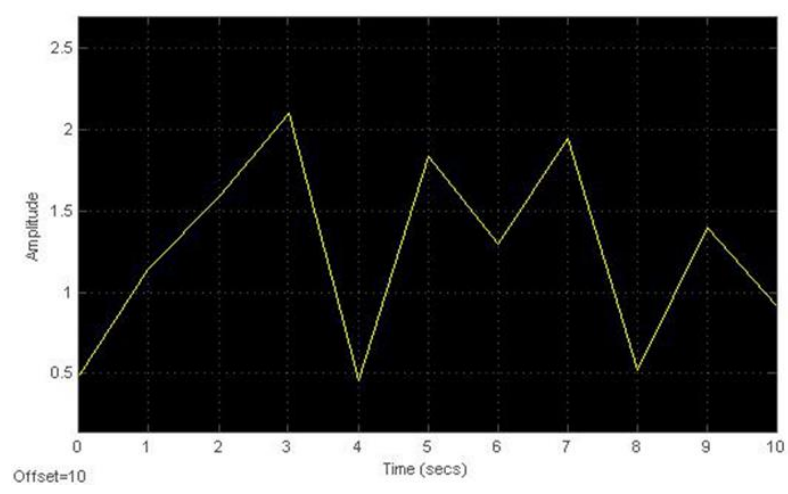

Figure 6. Rayleigh Noise Input

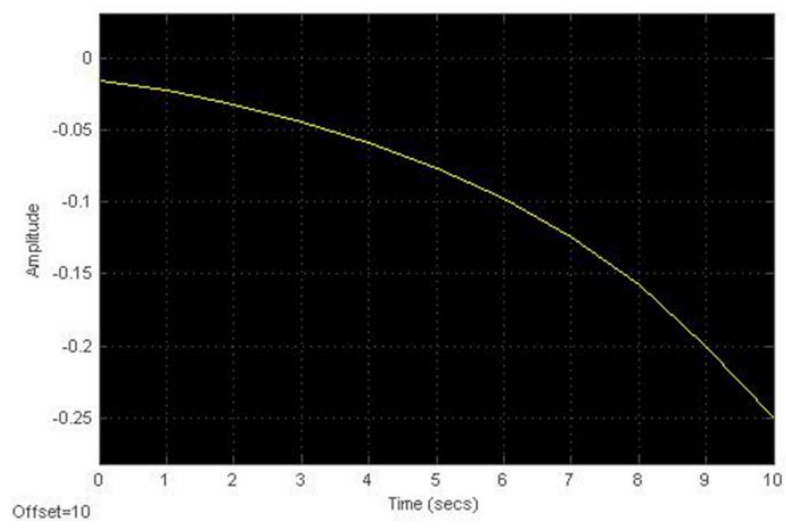

Figure 7. Rayleigh Noise Output

The waveform when observed at the output showed the desired results. The amplitude of the noise signal showed a considerable reduction and can be observed from the figures 4 and 5. A similar process was followed for noise signals separately. The source of the simulink model was replaced by a Band limited white noise generator instead of the mic and that a similar approach was followed for Rayleigh noise as well and the output for same can be observed in figure 7. Although the time scope 2 showed a better result than time scope 1(the input signal), the time scope showed the best result (final output). The approach of wavelet transform [6-7] cannot be ruled out to show better results in some cases, but the proposed algorithm also performed efficiently. The biquad filter when used alone did not display better results as it did after using a Hilbert filter in cascade. From figures 2 and 3, it can be observed that although the amplitude of the output signal has been reduced, the presence of noise signal has been reduced considerably. The latter statement can be verified from figures 5 and 6 . The peak of the Band limited white noise has been reduced substantially in figure 6 and the waveform has been smoothened due to filtering. Figures 7 and 8 also support the conclusion made in this paper for the same.

\section{Conclusion}

The model presented in this report can be used for removing any noise. The presented approach smoothens the noise and reduces its amplitude. The Hilbert transform is widely known for its noise removal features; cascading it with Biquad filter produced better results. However, better results can be obtained by using adaptive filtering for speech signals [9]. The work done in this paper is an analysis of both the filters and their role in removing noise from a particular category of signal. 


\section{Future Scope}

The work done in this paper can be further enhanced by the use of adaptive filters which might be efficient and provide better results in terms of noise removal and improved signal strength. Also, a deeper mathematical analysis of the model might pave way for optimal use of resources and a better extraction of the actual product.

\section{References}

[1] D. Juriši, N. Mijat and I. Mihali, "General Purpose Biquads Optimized for Dynamic Range and Low Noise", IEEE MELECON, (2006) May, pp. 31-34.

[2] P. Monsurrò, S. Pennisi, G. Scotti and A. Trifiletti, "Design Strategy For Biquad-Based ContinuousTime Low-Pass Filters", 20th European Conf. on Circuit Theory and Design, (2011) pp. 385-388.

[3] N. Madhu, A. Spriet, S. Jansen, R. Koning and J. Wouters, "The Potential for Speech Intelligibility Improvement Using the Ideal Binary Mask and the Ideal Wiener Filter in Single Channel Noise Reduction Systems: Application to Auditory Prostheses", IEEE Trans. Audio, Speech and Language Processing, Vol. 21, pp. 63-72, January 2013.

[4] S. Lei and R. Jansen, "PWM Based Sensing and Control of Magnetic Bearings", IPEMC 2006.

[5] E. Hermanowicz and A. Paruzel, "Farrow Structure For Complex Digital Hilbert Filter Of Low Complexity".

[6] H. Sepehr, M. Ahmadi and P. Brennan, "Removal Of Siren Mixed With Speech By Wavelet Transform And Adaptive Notch Filter", HSCMA 2008.

[7] R. J. Barsanti, J. Gilmore, "Comparing Noise Removal In The Wavelet And Fourier Domains”, pp 163167,2011

[8] N.W.Arshad, S.N.Abdul Aziz, F. Naim, R. Abdul Karim, R. Hamid and N. F. Zakaria," Speech Processing for Makhraj Recognition", 7th Int. Conf. on IT in Asia, 2011

[9] S. P. Nandyala and T. K. Kumar, "Speech Enhancement Using Kernel Adaptive Filtering Method", Dept. of Elec. and Comm. Engineering, NIT Warangal, India.

[10] Abhishek Katariya, Amita Yadav, Neha Jain, and Geetam Tomar, "BER Comparison of RS and BCH Coded OFDM Transmission Over NoisyChannel”, ”, IEEE International Conference on Computational Intelligence and Communication Networks CICN2011, pp 233- Oct 2011,

[11] C. H. Lin, J. S. Tsai and C. T. Chiu," Switching Bilateral Filter With A Texture/Noise Detector For Universal Noise Removal", ICASSP 2010, pp. 1434-1437.

[12] Programmable Double Biquad Filter for Tone Detection on Fixed Point DSPs, Texas Instruments, Application Report, SPRA482, Feb. 1999

\section{Author}

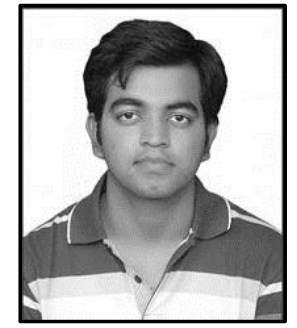

Animesh Kumar Jha, he received the B.Tech degree in Instrumentation and Control Engineering from Bharati Vidyapeeth's College of Engineering (GGSIPU, New Delhi) in 2013. He is currently working at Tata Consultancy Services, Mumbai as Product Specialist for Microsoft Dynamics CRM. His research interests include digital image processing, soft computing and robotics. 
International Journal of Signal Processing, Image Processing and Pattern Recognition Vol.8, No.9 (2015) 